\title{
Planning Oncologists of Ambulatory Care Units
}

\author{
Abdellah SADKI ${ }^{1}$, Xiaolan XIE ${ }^{1,3}$, Franck CHAUVIN ${ }^{2}$ \\ ${ }^{1}$ Center for Health Engineering and ROGI/LSTI \\ École Nationale Supérieure des Mines de Saint Etienne \\ 158 cours Fauriel, 42023 Saint Etienne, France \\ ${ }^{2}$ Institut de Cancérologie de la Loire \\ 108 av Albert Raimond, 42271 Saint-Priest-en-Jarez, France \\ ${ }^{3}$ Center for Health Care Engineering \\ Shanghai Jiao Tong University \\ 800 Dongchuan Road, Minhang, Shanghai 200240, China
}

\section{Corresponding author:}

Prof. Xiaolan XIE, Department of Healthcare Engineering, École Nationale Supérieure des Mines de Saint Etienne, 158 Cours Fauriel, 42023 Saint Etienne, France. Tel: +33 4774266 95. Fax: +33 477 4202 49. Email: xie@emse.fr

\begin{abstract}
This paper addresses the problem of determining the work schedule, called medical planning, of oncologists for chemotherapy of oncology patients at ambulatory care units. A mixed integer programming (MIP) model is proposed for medical planning in order to best balance bed capacity requirements under capacity constraints of key resources such as beds and oncologists. The most salient feature of the MIP model is the explicit modeling of specific features of chemotherapy such as treatment protocols. The medical planning problem is proved to be NP-complete. A threestage approach is proposed for determining good medical planning in reasonable computational time. From numerical experiments based on field data, the three-stage approach takes less than 10 minutes and always outperforms the direct application of MIP solvers with 10h CPU time. Compared with the current planning, the three-stage approach reduces the peak daily bed capacity requirement by $20 \mathrm{~h}$ to $45 \mathrm{~h}$ while the maximum theoretical daily bed capacity is $162 \mathrm{~h}$.
\end{abstract}

Keywords: Oncology, ambulatory care, medical planning, bed capacity, mixed integer programming. 


\section{INTRODUCTION}

This research is performed in close collaboration with the cancer center ICL. The « Institut de Cancérologie de la Loire» (Loire Cancer Institute), a.k.a. ICL, is a French public comprehensive cancer center providing oncology, hematology, pediatric oncology and radiotherapy services. ICL is also the linchpin of the Oncoloire network, which is the care network for cancer patients and aims to ensure that all cancer patients in the Loire department can be treated equally regardless of where they live. In 2008 ICL delivered: 12000 chemotherapy sessions, 10679 hospitalization days in oncology for 1843 direct entrances, 5623 days of hospitalization in hematology for 411 direct entrances, 2361 days of hospitalization in pediatric oncology for 454 direct entrances and 39266 radiotherapy sessions for 1706 patients. ICL provides an academic teaching and research environment; it serves to train medical students and interns from many disciplines. Also, numerous patients participate in clinical trials. All this complicate the center resource planning.

This paper addresses the medical planning problem of oncologists at an ambulatory care unit (ACU), i.e. the determination of their work schedule and also called medical planning, in order to balance the bed capacity requirement over time while taking into account treatment protocols of patients. The problem was brought to our attention by the ICL ambulatory care unit which provides chemotherapy treatment for outpatients in oncology and is common to most oncology ambulatory care units in France. As the demand for oncology cares increases, ICL faces serious capacity problem. The ACU unit is often overcrowded and the ACU staff was asking the management to increase the number of beds. We were asked to assess the bed requirement of the ACU unit. Data collected from field show that the actual daily bed capacity requirement (see Figure 1) is highly unbalanced. This observation motivates the research presented in this paper for smoothing the daily bed capacity requirement in order to avoid the bed crises of the ACU unit.

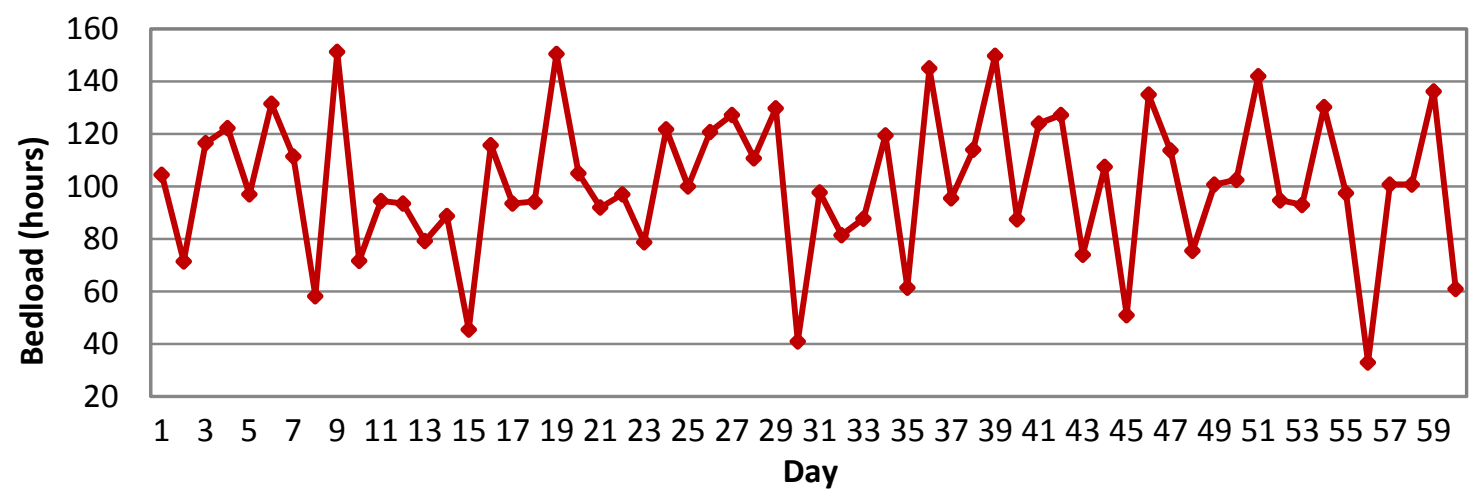

Figure 1: Actual daily bed capacity requirement

To the best of our knowledge, this problem has not been addressed in the literature. This paper pursues our preliminary work (Mazier and Xie[13]) which shows that peak bed capacity requirement can be 
reduced by standard optimization solvers provided that computation time over $10 \mathrm{~h}$ and sometime over 1 days is acceptable.

In this paper, we first propose a mixed integer programming (MIP) model that takes into account obvious capacity constraints of resources such as beds needed during chemotherapy and consultation capacity of oncologists. The most important feature of the MIP model is the explicit modeling of patient treatment protocols which gives a precise estimation of resource requirement of patients over time. The medical planning problem is proved to be NP-complete and it takes standard MIP solvers over $10 \mathrm{~h}$ to determine acceptable solutions. We then propose a three-stage approach which first determines the morning medical planning, then the afternoon medical planning with the given morning medical planning, and local search for improvement of the resulting medical planning. Numerical experiments with field data show that the three-stage approach takes less than 10 minutes CPU time and always outperforms the direct application of MIP solvers to the MIP model with 10h CPU time. Further, the three-stage approach outperforms the current planning in both bed capacity requirement balancing and extra consultation capacity needed. Over 10 problem instances derived from field data, the peak bed capacity requirement is reduced by $20 \mathrm{~h}$ to $45 \mathrm{~h}$ per day while the theoretical maximum bed capacity is $162 \mathrm{~h}$ per day.

The literature on operation management of oncology care centers is scarce. Conforti et al [5] proposed an optimization model for outpatient scheduling within a radiotherapy department in order to minimize waiting time and waiting lists for radiotherapy treatments. Santibáñez et al [15] used simulation to analyze the impact of operations, scheduling and resource allocation on patient waiting time, clinic overtime, and resource utilization at British Columbia Cancer Agency's ambulatory care unit. Blay et al [2] conducted a three-month work sampling to evaluate the roles and workload of nurses within an outpatient oncology unit. Nurses were found to have a large administrative role while their nursing activities range from basic nursing tasks to more specialist activities including patient counseling and complex chemotherapy regimens. Delaney et al [6] performed a multivariate analysis of the chemotherapy treatment duration based on patient -tumor- and treatment related factors. Significant factors that impacted on treatment duration were the chemotherapy regimen, type of infusion, and patient age. Wide fluctuations of workload were observed while the daily number of patients remained stable. Matta and Patterson [12] developed a stratification framework and an evaluation construct to address the problem of multiple responses in simulation experiments of outpatient clinics. This approach was used to evaluate initiatives for operational performance improvement of a large oncology center.

This paper is also related to the general area of appointment scheduling or outpatient scheduling for which is a rich literature is available. Cayirli and Veral [3] provided a comprehensive review of appointment scheduling in outpatient services. Gupta and Denton [8] summarized key issues in design- 
ing and managing patient appointment systems for health services in order to clarify the level of complexity encountered in the health care environment. Taxonomy of complicating factors was provided. Bailey [1] focused on patient waiting times of scheduling appointment rules. Cayirli et al [4] used simulation to evaluate patient and doctor-related performance measures of different appointment rules. They showed that patient sequencing has a greater effect on ambulatory care performance than the choice of an appointment rule, and that panel characteristics influence the effectiveness of appointment systems. Liu and Liu[10], [11] performed a specific queuing analysis of appointment systems with multiple physicians and random late arrival of physicians. Klassen and Rohleder [9] compared various scheduling rules in order to minimize the waiting time of patients as well as the idle time of doctors. Walczak et al [17] used neural network to design a decision support tool for allocating hospital beds and determining required acuity of care. Schaus and Hentenryck [16] considered the daily assignment of newborn infant patients to nurses in a hospital in order to balance the workload of the nurses. Constraint programming was used to solve this problem in two steps: assignment of nurses to zones and assignment of nurses to patients.

There are several major differences between medical planning of oncologists and existing appointment scheduling models. Oncology patients have to follow specific treatment protocols which lead to complex dynamic variations for bed capacity requirement over time. A patient assigned a chemotherapy weekday will always come the same weekday for different chemotherapy sessions over the treatment protocol. Finally, each oncology patient is assigned a referee oncologist for better follow-up and the patient should be assigned a day for chemotherapy when his referee oncologist works. These specific features of oncology cares make the medical planning of oncologists different from existing appointment scheduling models.

The rest of the paper is organized as follows. Section 2 describes the chemotherapy process at an ACU. Section 3 presents assumptions of our medical planning model, provides a formal mixed integer programming formulation and proves the complexity of the problem. Section 4 proposes a three-stage approach. Section 5 presents numerical results to assess the benefits of medical planning optimization and the performance of the three-stage approach. Section 6 is a conclusion.

\section{CHEMOTHERAPY PROCESS}

Cancer disease is destructive and in many cases fatal. Chemotherapy has shown success in treating the disease. Chemotherapy uses drugs to kill cancer cells and to prevent them from growing and multiplying. The type of chemotherapy treatment of a patient is determined after an initial consultation with an oncologist and the assessment by a team of oncologists. Chemotherapy is given in several ways: intravenously, orally, through an injection, or topically (applied on the skin). The most common method of 
delivering chemotherapy is intravenously and the procedures range from 15 minutes to 7 hours or longer. These variations complicate appointment scheduling and bed allocation.

The chemotherapy is often given in cycles that include treatment sessions separated by rest periods. The length of each cycle and the number of cycles in the treatment plan are defined in a protocol according to the type of cancer.

Table 1 gives some examples of protocols. With Avastin, a patient receives the chemotherapy injection once every two weeks. With Cisplantin, a patient receives chemotherapy in weeks 1,2 and 4 but not in week 3 , The treatment repeats over several months as needed. A patient always comes the same weekday for different treatment sessions in order to respect the treatment protocol.

Table 1: Chemotherapy protocols

\begin{tabular}{|c|c|c|c|c|}
\hline \multirow{2}{*}{ Protocol } & \multicolumn{4}{|c|}{ Periodicity } \\
\cline { 2 - 5 } & Week1 & Week2 & Week3 & Week4 \\
\hline Avastin & 1 & 0 & 1 & 0 \\
\hline Cisplatin & 1 & 1 & 0 & 1 \\
\hline Rituximab & 1 & 0 & 0 & 1 \\
\hline Vinorelbin & 1 & 1 & 0 & 0 \\
\hline
\end{tabular}

Every patient is assigned a referee oncologist who ensures the follow-up of the treatment. Hence, a patient has to come a day when his referee oncologist works. If necessary, the patient can be seen by another oncologist or an intern.

Each chemotherapy session on a day $D$ follows the following process. On day $D-1$, blood tests are performed often by an outside medical analysis laboratory in order to determine whether the patient is in a good enough health condition to receive the chemotherapy. The two most important measures in these blood tests are blood counts and liver health. It is important that the liver be healthy, as it will be needed to help remove the chemotherapy drugs from the body. Blood counts indicate whether the immune system is healthy enough to help heal cells that may be damaged during the chemotherapy process. Results of blood tests are sent to the ACU the day before the chemotherapy by the laboratory.

On the day $D$ of chemotherapy, the treatment process of a patient is illustrated by Figure 2. Upon his arrival at the hospital, the patient checks in at the reception, goes to the waiting room and stays there until the referee physician is available. The consultation takes place in a consultation box. Depending on the results of blood test and patient's health condition, the physician then decides on whether the patient can receive the chemotherapy. If not, the physician either postpones the treatment session for some days (one week in most case) or alters the treatment protocol. If the patient is in good health condition, the physician prescribes medicines of the chemotherapy and the prescription is sent to the pharmacy to initiate the preparation of the chemotherapy drugs. The patient returns to the waiting 
room after the consultation. When a bed becomes available, an ACU nurse places the patient in a bed and prepares the patient for injection. Once the drug is ready and the patient has been prepared, the injection starts. After the injection of the corresponding chemotherapy drugs, the patient is given appointment for his next chemotherapy session and leaves the hospital.

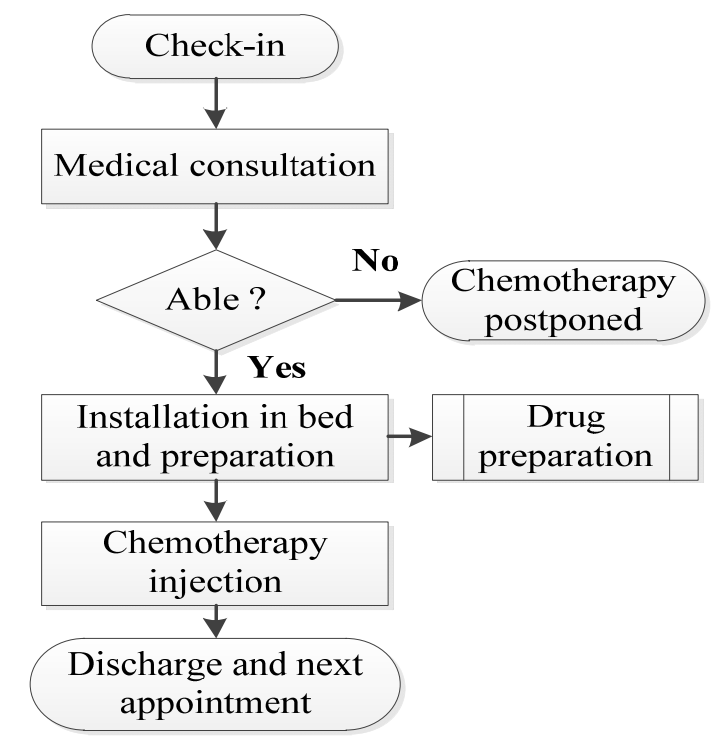

Figure 2 : Care process of a chemotherapy session

For an oncology Ambulatory Care Unit (ACU), major operation decisions include: (i) medical planning once a year to determine the working periods of oncologists, (ii) patient assignment which determines the day of chemotherapy for each incoming patient; (iii) appointment scheduling which determines the appointment time for the next chemotherapy session of each patient; (iv) the production scheduling of chemotherapy drugs. This paper focuses on medical planning.

\section{PROBLEM SETTING AND COMPLEXITY}

This section provides a formal description of the medical planning problem in order to best balance the bed capacity requirement over time. We first present assumptions of the medical planning model, then propose a mixed integer programming formulation, and analyze the complexity of the problem.

\subsection{Assumptions}

Some assumptions are needed in order to simplify the problem.

Assumption A1. Only the weekday of chemotherapy of a patient is determined and the exact appointment time is not considered. 
Assumption A2. The preparation of the drugs at the pharmacy is not considered and the chemotherapy drugs for a patient are assumed ready when a bed is available for the patient and the patient is prepared for injection.

Assumption A3. Enough ACU nurses are available. The preparation of a patient can start when a bed becomes available and the injection starts after the preparation.

Assumption A4. Each chemotherapy session can start and finish in the same day.

Assumption A3 is reasonable as the ACU is usually staffed according to the standard nurse to bed ratio. Assumption A1 is natural with regard to the medical planning problem which is a long term decision made yearly or when major change occurs. Assumption A2 is a consequence of Assumption A1. Further, by better balancing the bed capacity requirement at the $\mathrm{ACU}$, it is expected that the workload of the nurses will be better balanced. This, together with appropriate patient appointment scheduling that is not addressed in this paper, will allow better synchronization between the drug preparation and the patient treatment. Assumption A4 excludes chemotherapies longer than a day. This is natural as an outpatient unit opens only during the day and chemotherapies with longer injection times are performed in inpatient units.

Three major resources are considered: physicians, beds, and consultation boxes. Physicians include oncologists and interns. A limited number of consultation boxes are available for oncologists. The consultation boxes for interns are not considered. Bed capacity is important as a bed is needed during the injection of the chemotherapy drug and the injection time is very long.

An ACU is open five days a week (Monday to Friday). Each day is divided into two periods (morning and afternoon). Each period is characterized by the number of consultation boxes available and the number of patients an oncologist can consult. There is only one intern in the ICL-ACU who is always available and can consult patients for all oncologists under their supervision. However the approaches proposed in this paper can be easily extended to the general case with any number of interns. The approaches also apply if the intern can only consult patients of some physicians.

Assumption A5. A time horizon of $W$ weeks is considered and the set $P$ of patients treated in this horizon is assumed known. Each patient starts the chemotherapy at a given week according to a given protocol with a given referee oncologist.

This is actually a very important assumption. Practically, we consider patients treated during a period of time in the past and try to optimize the medical planning and patient assignment with respect to this 
set of patients. As the medical planning will be applied for the future, the key question is the goodness of such an approach with respect to unknown future patient flow. This issue is addressed in a separate study [14] which shows that the medical planning derived from historical data, together with an efficient patient assignment strategy, remains efficient with respect to unknown future patient flow.

Under Assumption A5, each patient $i$ is also assigned to a referee oncologist and the set $P$ of patients can be partitioned into disjoint subsets of patients, $P=\bigcup_{j \in J} P_{j}$, where $P_{j}$ denotes the set of patients of oncologist $j$ and $J$ is the set of oncologists.

Chemotherapy sessions of a patient $i$ can be represented by a $W$-dimension binary vector $\boldsymbol{a}_{i}$ based on the treatment protocol and the arrival date of the patient. An entry $a_{i w}$ is equal to 1 if patient $i$ requires a chemotherapy session in week $w, a_{i w}=0$ otherwise. Of course, for a patient $i$ starting its treatment plan on week $w_{0}$ and ends its treatment plan on week $w_{1}, a_{i w}=0$, for all $w<w_{0}$ or $w>w_{1}$. All other entries are determined according to the corresponding protocol. Table 2 gives some examples of treatment sessions of different patients. For example, patient 3 is treated from week 3 to week 7 according to the Avastin protocol.

Table 2: Chemotherapy sessions over 8 weeks

\begin{tabular}{|c|c|c|c|c|c|c|c|c|c|c|c|}
\hline patient & start & end & protocol & week1 & week2 & week3 & week4 & week5 & week6 & week7 & week8 \\
\hline 1 & 1 & 3 & Avastin & 1 & 0 & 1 & 0 & 0 & 0 & 0 & 0 \\
\hline 2 & 3 & 7 & Avastin & 0 & 0 & 1 & 0 & 1 & 0 & 1 & 0 \\
\hline 3 & 1 & 16 & Cisplatin & 1 & 1 & 0 & 1 & 1 & 0 & 1 & 1 \\
\hline 4 & 2 & 7 & Vinorelbin & 0 & 1 & 1 & 0 & 0 & 1 & 1 & 0 \\
\hline
\end{tabular}

Assumption A6. Each day is decomposed into two time periods: morning and afternoon. A patient can be consulted either in the morning or in the afternoon. A chemotherapy session started in the morning can finish in the afternoon.

Assumption A6 follows the current practice of most cancer centers. Of course shorter time periods can be considered with obvious increasing complexity of the problem.

\subsection{Mixed integer programming model}

Under Assumptions A1-A6, the medical planning problem is characterized mainly by a set of oncologists, interns, a planning horizon of $W$ weeks, a set of patients with for each patients a treatment protocol and a referee oncologist. Other relevant data include: consultation capacity, beds, injection times and consultation boxes available that will be described later. The objective is to best balance the bed capacity requirement over the planning horizon. 
Four sets of decisions need to be made. The first one is the so-called medical planning which indicates for each oncologist the time periods he consults. The medical planning is denoted by binary variables $y_{j t}$ with

$$
y_{j t}=\left\{\begin{array}{l}
1, \text { if oncologist } j \text { consults in period } t . \\
0, \text { otherwise }
\end{array}\right.
$$

for all oncologists $j \in J$ and for all time periods $t \in T$ where $T$ is the set of the ten time periods in a week including morning and afternoon periods of all weekdays. The same medical planning is applied for different weeks over the planning horizon. This is natural as the oncology protocols require each patient to come the same weekday in different weeks for different treatment sessions.

The second set of decisions concerns the patient assignment which indicates which time period of the week a patient comes for the chemotherapy. Patient assignment is denoted by binary variables $x_{i t}$ with

$$
x_{i t}=\left\{\begin{array}{l}
1, \text { if patient } i \text { is consulted in period } t \\
0, \text { otherwise }
\end{array}\right.
$$

for all patients $i \in P$ and for all $t \in T \cdot x_{i t}=1$ also implies that the chemotherapy of the patient starts in period $t$. In this paper, we do not postpone the start of the treatment plan decided by oncologists but select the day patient comes for chemotherapy.

According to the treatment protocols, each patient $i$ comes in time period $t$ of week $w$ if $a_{i w}=1$ and $x_{i t}$ $=1$. Note that, due to the length of chemotherapy, a chemotherapy session started in the morning can finish in the afternoon of the same day. We do not consider chemotherapy sessions that last for more than one day. Such treatments are not performed in an ACU.

The third set of decisions concerns patients consulted by interns. The non negative integer variable $z_{j w t}$ denotes the number of patients of oncologist $j$ consulted by interns in period $t$ of week $w$.

The last set of decisions concerns the extra consultation capacity needed for each period. The nonnegative integer variable

$$
e_{j w t}
$$

denotes the number of patients of oncologist $j$ consulted in period $t$ of the week $w$ with extra consultation capacity.

The decisions should be made under the following constraints. First, the number of oncologists that consult in period $t$ should not exceed the number $B_{t}$ of consulting boxes available, i.e.

$$
\sum_{j \in J} y_{j t} \leq B_{t}, \forall t \in T
$$


Each patient should be assigned a time period, i.e.

$$
\sum_{t \in T} x_{i t}=1, \quad \forall i \in P
$$

The number of patients that come a period in any week should not exceed the consultation capacity of the oncologists, the interns and the extra consultation capacity. More specifically,

$$
\begin{gathered}
\sum_{i \in P_{j}} a_{i w} x_{i t} \leq N_{t} y_{j t}+z_{j w t}+e_{j w t}, \quad \forall j \in J, t \in T, w \in W \\
\sum_{j \in J} z_{j w t} \leq N_{t}^{\prime}, \quad \forall t \in T, w \in W
\end{gathered}
$$

where $N_{t}$ is the maximum number of patients an oncologist can consult in period $t, N_{t}^{\prime}$ is the maximal number of patients the interns can consult. Note that, for simplicity, the same consultation capacity is assumed for all oncologists. Extension to different consultation capacity is trivial.

In order to ensure the possibility of each patient to be seen by his referee oncologist, it is assumed that each patient should be assigned a time period where the referee oncologist consults. This is ensured jointly by the above consultation capacity constraints and the following constraints that forbid the delegation of patients of oncologist $j$ and the use of extra consultation capacity if he does not consult in period $t$.

$$
z_{j w t}+e_{j w t} \leq\left|P_{j}\right| y_{j t}, \quad \forall j \in J, t \in T, w \in W
$$

This constraint can also be replaced by the following equivalent constraints:

$$
x_{i t} \leq y_{j t}, \quad \forall j \in J, i \in P_{j}, t \in T
$$

Another constraint is the bed capacity of the afternoon periods which is ensured jointly by two constraint sets. The first forbid long chemotherapies that cannot start and finish in the afternoon. More specifically,

$$
x_{i t}=0, \forall i \in P / d_{i} \geq L_{t}, t \in P M
$$

where $d_{i}$ is the time patient $i$ spent in a bed for a treatment session including injection time and patient preparation time, $L_{t}$ is the ACU opening time during the afternoon, $P M$ is the set of afternoon time periods. A second constraint restricts the total bed capacity requirement of chemotherapy sessions starting in the afternoon. More specifically,

$$
\sum_{i \in P} a_{i w} d_{i} x_{i t} \leq Q_{t}, \quad \forall w \in W, t \in P M
$$

where $Q_{t}$ is the maximum bed capacity of the afternoon, i.e. $Q_{t}=l \times L_{t}$ where $l$ is the number of beds available in the ACU. Such constraints are not considered for morning time periods as chemotherapy sessions started in the morning can finish in the afternoon. 
In this paper, $d_{i}$ is called injection time for simplicity. Further, it is assumed to be a positive integer. Practically, all injections times are expressed as integer multiples of some base time unit $\Delta$. In our numerical experiments, $\Delta=15$ minutes.

The goal is to best balance the bed capacity requirement during different weeks and to use as less as possible extra consultation capacity. More specifically,

$$
\operatorname{MIN} \sum_{w \in W}\left(\bar{C}_{w}-\underline{C}_{w}\right)+M \sum_{j \in J} \sum_{w \in W} \sum_{t \in T} e_{j w t}
$$

where $M$ is a positive integer serving as weighting factor for extra consultation capacity, $\bar{C}_{w}$ and $\underline{C}_{w}$ denote respectively the maximum and minimum bed capacity requirements of week $w$ with

$$
\begin{array}{ll}
\sum_{i \in P} a_{i w} d_{i}\left(x_{i t}+x_{i t+1}\right) \leq \bar{C}_{w}, & \forall w \in W, t \in A M \\
\sum_{i \in P} a_{i w} d_{i}\left(x_{i t}+x_{i t+1}\right) \geq \underline{C}_{w}, & \forall w \in W, t \in A M
\end{array}
$$

where $A M$ is the set of morning time periods and $\left(x_{i t}+x_{i t+1}\right)$ indicates whether patient $i$ is treated in the day corresponding to morning period $t$. As each patient is assigned to only one period $t$, $\sum_{i \in P} a_{i w} d_{i}\left(x_{i t}+x_{i t+1}\right)$ gives the bed capacity requirement for the corresponding day in week $w$. Note that an alternative approach to our single criterion approach is to apply multi-criteria approaches for bed capacity requirement balancing and extra consultation capacity minimization..

The mathematical model of the medical planning problem described above is a mixed integer programming problem summarized as follows:

MIP: $\quad J^{*}=\operatorname{MIN} \sum_{w \in W}\left(\bar{C}_{w}-\underline{C}_{w}\right)+M \sum_{j \in J} \sum_{w \in W} \sum_{t \in T} e_{j w t}$

subject to:

$$
\begin{aligned}
& \sum_{t \in T} x_{i t}=1, \quad \forall i \in P \\
& \sum_{j \in J} y_{j t} \leq B_{t}, \quad \forall t \in T \\
& \sum_{i \in P_{j}} a_{i w} x_{i t} \leq N_{t} y_{j t}+z_{j w t}+e_{j w t}, \quad \forall j \in J, t \in T, w \in W \\
& \sum_{j \in J} z_{j w t} \leq N^{\prime}, \quad \forall t \in T, w \in W \\
& z_{j w t}+e_{j w t} \leq\left|P_{j}\right| y_{j t}, \quad \forall j \in J, t \in T, w \in W
\end{aligned}
$$




$$
\sum_{i \in P} a_{i w} d_{i} x_{i t} \leq Q_{t}, \quad \forall w \in W, t \in P M
$$

$$
\sum_{i \in P} a_{i w} d_{i}\left(x_{i t}+x_{i t+1}\right) \leq \bar{C}_{w}, \quad \forall w \in W, t \in A M
$$

$$
\sum_{i \in P} a_{i w} d_{i}\left(x_{i t}+x_{i t+1}\right) \geq \underline{C}_{w}, \quad \forall w \in W, t \in A M
$$

$$
x_{i t}=0, \forall i \in P / d_{i} \geq L_{t}, t \in P M
$$

$$
x_{i t}, y_{j t} \in\{0,1\}, e_{j w t}, z_{j w t} \geq 0
$$

Compared to our previous work [13], the above MIP formulation aims at smoothing the bed capacity requirement of each week instead of smoothing the bed capacity requirement over the whole planning horizon. Given the patient arrivals and patient treatment plan characterized by $a_{i w}$, the total weekly bed capacity requirements are given constants $\sum_{i \in P} a_{i w} d_{i}$. As a result, the new MIP formulation seems more reasonable and takes into account given variations of week capacity requirement. Another new ingredient of the new MIP formulation is the relaxation of the consultation capacity constraint and hence, the new MIP model has at least one solution.

Given the mathematical formulation, a straightforward method for medical planning is to solve directly the mixed integer programming model MIP using standard optimization engines. Unfortunately this turns out to be highly time consuming. It takes CPLEX solver over $10 \mathrm{~h}$ to provide an acceptable solution for problem instances related to our case study. This is confirmed by the following complexity result.

Theorem 1: The medical planning problem is NP-complete.

The proof of the theorem is given in the Appendix. The computation time of over 10 hours is too long for real life application in oncology ACUs and efficient optimization methods are needed.

Theorem 2: The MIP model (1)-(11) has a solution only if $\sum_{t \in T} B_{t} \geq|J|$ and it has a solution if $\sum_{t \in A M} B_{t} \geq|J|$. Further, any medical planning $\left\{y_{j t}\right\}$ in which at least one oncologist does not have consultation slots is not feasible.

Proof: From constraints (2), (4) and (6), the MIP model has a solution only if each oncologist can be assigned either at least one time period for consultation, which proves the second part of the theorem. Combining it with the number of consultation boxes, $\sum_{t \in T} B_{t} \geq|J|$ and the necessary condition 
holds. If $\sum_{t \in A M} B_{t} \geq|J|$, each oncologist can be assigned a morning time period for consultation. Assigning all patients of each oncologist to his morning consultation time period leads to a feasible solution and concludes the proof. Q.E.D.

\section{A THREE-STAGE APPROACH FOR MEDICAL PLANNING}

This section proposes a three-stage approach. It relies on our observation that the MIP model can be quickly solved if the medical planning is given. The first stage determines the medical planning for morning time periods. The second stage determines medical planning for afternoon periods by fixing the morning medical planning. The resulting medical planning is then improved with local optimization in the third stage. In order to limit the computational time, the two first stages are based on a subset $W^{\prime} \subset W$ of weeks of the highest weekly bed capacity requirement, i.e. the highest $\sum_{i \in P} a_{i w} d_{i}$.

\subsection{Morning medical planning}

The first stage of our approach determines the medical planning for the morning periods $t \in A M$. As only morning periods are considered, it is not possible to assign all patients and constraints (2) of MIP model on the assignment of all patients are relaxed as follows:

$\sum_{t \in T} x_{i t} \leq 1, \quad \forall i \in P$

Extra consultation capacity is not allowed. The objective of this stage is to insert as many patients as possible and to best balance the daily bed capacity requirement. More specifically, the first stage solves the following mixed integer programming problem:

$\operatorname{MIP}^{\mathrm{AM}}: \operatorname{MIN} \sum_{w \in W^{\prime}}\left(\bar{C}_{w}-\underline{C}_{w}\right)+M^{\prime} \sum_{i \in P}\left(1-\sum_{t \in A M} x_{i t}\right)$

subject to constraints (12), (3)-(6) and (11) with $e_{j w t}=0, T$ replaced by the set $A M$ of morning periods and $W$ replaced by the set $W^{\prime}$ of selected weeks and

$$
\begin{array}{ll}
\sum_{i \in P} a_{i w} d_{i} x_{i t} \leq \bar{C}_{w}, & \forall w \in W^{\prime}, t \in A M \\
\sum_{i \in P} a_{i w} d_{i} x_{i t} \geq \underline{C}_{w}, & \forall w \in W^{\prime}, t \in A M
\end{array}
$$

The above criterion (13) is a combination of two criteria: daily bed capacity requirement balancing $\sum_{w \in W^{\prime}}\left(\bar{C}_{w}-\underline{C}_{w}\right)$ and the number $\sum_{i \in P}\left(1-\sum_{t \in A M} x_{i t}\right)$ of unassigned patients. $M^{\prime}$ is a large constant in order to insert as many patients as possible. 


\subsection{Afternoon medical planning}

The morning medical planning being given, the second stage extends the morning medical planning formulation to schedule afternoon consultation time slots. Both morning and afternoon time periods are considered except that the medical planning of the morning time periods is fixed to that determined at stage 1. Each patient can now be assigned to a morning period, an afternoon period or unassigned. More specifically, stage 2 solves the following mixed integer programming problem:

$\operatorname{MIP}^{\mathrm{PM}}: \operatorname{MIN} \sum_{w \in W^{\prime}}\left(\bar{C}_{w}-\underline{C}_{w}\right)+M^{\prime} \sum_{i \in P}\left(1-\sum_{t \in T} x_{i t}\right)$

subject to constraints (12), (3)-(6) and (11) with $e_{j w t}=0, W$ replaced by the set $W^{\prime}$ of selected weeks and the morning medical planning $\left\{y_{j t} \forall t \in A M\right\}$ being that determined in stage 1, plus (7)-(10) to include constraints related to afternoon medical planning. As in stage 1, stage 2 tries to insert as many patients as possible with respect the complete medical planning of both morning and afternoon. The criterion (16) is similar to criterion (13).

Note that the afternoon medical planning could also be determined using the MIP model with morning medical planning being given. Numerical experiments show that the two approaches leads to similar results. However, the $M I P^{P M}$ is less time consuming as continuous variables $e_{j w t}$ are not considered.

\subsection{Local optimization of the medical planning}

Starting from the complete medical planning determining in stages 1 and 2, the third stage iteratively improves the medical planning by local search and an approximation scheme for quick evaluation of local solutions.

A medical planning $\left\{y_{j t} \forall t \in T\right\}$ can be equivalently represented by a subset of oncologists assigned to each time period. Each time period $t$ has $B_{t}$ consultation boxes. We call a consultation time slot the combination of a time period and a consultation box. Hence there are $B_{t}$ consultation time slots in period $t$. To each medical planning is associated an assignment of consultation time slots to oncologists. Any unused consultation time slot is assumed to be assigned to a fictitious oncologist in order to have a complete assignment. Hence a medical planning can be represented by a $\sum_{t \in T} B_{t}$ dimension vector with each entry denoting the oncologist assigned to the corresponding consultation time slot.

The neighborhood of a given medical planning contains the set of all medical planning obtained by one of the two local moves:

(i) interchange the oncologist assignment of two consultation time slots belonging to two different time periods and assigned to two different oncologists. One of the oncologists could be fictitious. 
More specifically, we replace an oncologist assignment $\left(i, t_{i}\right)$ and $\left(j, t_{j}\right)$ for oncologists $i$ and $j$ by assignment $\left(j, t_{i}\right)$ and $\left(i, t_{j}\right)$;

(ii) give a consultation time slot of an oncologist to another oncologist such that the latter is either fictitious or not already given another time slot in the same time period. More specifically, we replace an oncologist-time slot assignment $(i, t)$ by $(j, t)$ where $i$ and $j$ are oncologists and $t$ a consultation time slot.

Move (ii) changes the number of consultation time slots assigned to each oncologist while move (i) modifies the schedule. It can be shown that any medical planning can be reached from another medical planning by a series of local moves.

The starting point of the local search is the medical planning $y^{0}$ determined in stages $1-2$. Its quality is evaluated by solving the MIP model by fixing the medical planning. Let $M I P\left(y^{0}\right)$ be the corresponding criterion value.

Starting from $y^{0}$, the local search algorithm determines all neighbor solutions $y$ of $y^{0}$. Let $N H\left(y^{0}\right)$ be the set of neighbor solutions of $y^{0}$. The neighborhood is large with about 300 neighbors in our numerical experiments and solving the MIP model exactly for each neighbor solution $y$ in order to identify the best neighbor solution is very time consuming. Instead, linear relaxation is used to identify the most promising neighbor.

More specifically, for each neighbor solution $y$, we first check whether the linear relaxation of the system defined by (2)-(11) with the integrity of $x_{i t}$ relaxed and

$$
\sum_{w \in W}\left(\bar{C}_{w}-\underline{C}_{w}\right)+M \sum_{j \in J} \sum_{w \in W} \sum_{t \in T} e_{j w t} \leq M I P\left(y^{0}\right)-1
$$

has a solution. Constraint (17) is introduced to check whether there exists a relaxed solution with medical planning $y$ that has a criterion value (1) smaller or equal to $M I P\left(y^{0}\right)-1$. As injection times $d_{i}$ and weighting factor $M$ are all assumed to be integers, the optimal criterion value $M I P(y)$ of the MIP model (1)-(11) for any medical planning $y$ is also an integer. Hence the linear relaxation of the system defined by (2)-(11) and (17) for medical planning $y$ provides a necessary condition for $y$ to be strictly better than $y^{0}$, i.e. $M I P(y) \leq M I P\left(y^{0}\right)-1$.

If the relaxed system does not has a solution, then $\operatorname{MIP}(y) \geq M I P\left(y^{0}\right)$ and the medical planning $y$ is not strictly better than $\operatorname{MIP}\left(y^{0}\right)$. Otherwise, the linear relaxation of MIP model (1)-(11) with the given medical planning $y$ is solved. Let $\operatorname{LBMIP}(y)$ be the corresponding criterion value which is a lower bound of the exact criterion of the medical planning $y$. From (17), LBMIP(y) $\leq M I P\left(y^{0}\right)-1$. 
The neighbor solution $y^{l}$ with the smallest lower bound $L B M I P(y)$ is selected. MIP model is then solved exactly for medical planning $y^{l}$ to obtain the exact criterion value $\operatorname{MIP}\left(y^{l}\right)$. If $\operatorname{MIP}\left(y^{l}\right)<$ $\operatorname{MIP}\left(y^{0}\right)$, the local search continues with $y^{l}$. Otherwise, the local search stops

The overall optimization method can be summarized as follows:

\section{MIP-based physician schedule heuristic:}

Step 1: Select the $K$ busiest weeks $W^{\prime}$;

Step2: Solve $M I P^{A M}$ to determine the morning medical planning;

Step3: Solve $M I P^{P M}$ to determine the afternoon medical planning. Let $y^{0}$ be the resulting complete medical planning;

Step 4: Solve $M I P$ with the complete planning horizon with medical planning $y^{0}$. Let $M I P\left(y^{0}\right)$ be the resulting criterion value;

Step 5: Let $L B^{*}=\infty$;

Step 6: For each medical planning $y$ obtained by any of the two local moves that meets the feasibility conditions of Theorem 2,

6.1: If the linear relaxation of the system defined by (2)-(11) and (17) does not have a solution, then go to next medical planning;

6.2: Solve the linear relaxation of $M I P$ model with medical planning $y$. Let $L B M I P(y)$ the criterion value;

6.3: If $L B^{*}>\operatorname{LBMIP}(y)$, then $L B^{*}=\operatorname{LBMIP}(y)$ and $y^{l}=y$.

Step 7: If $L B^{*}=\infty$, stop; otherwise, solve MIP for medical planning $y^{l}$.

Step 8: If $M I P\left(y^{l}\right)<M I P\left(y^{0}\right)$, set $y^{0}:=y^{l}$ and go to Step 5. Otherwise, stop.

In our numerical experiments, $K=4$ busiest weeks are used in Stages 1 and 2. Further, a stopping criterion of a CPU time of 5 minutes and a gap of $2 \%$ is used in $M I P^{A M}$ and $M I P^{P M}$. The evaluation of each medical planning $M I P(y)$ is implemented with a stopping criterion of a CPU time of 1 minute.

\section{NUMERICAL RESULTS}

This section presents numerical results based on data collected from the field to evaluate the effectiveness of the three-stage approach with respect to current practice. 


\subsection{Experimental setting}

The numerical experiments of this paper are based on field data collected from the ambulatory care unit of ICL for the year 2008.

The ACU has 10 oncologists and one intern. It has 18 beds for oncology patients. With a maximum possible utilization of $9 \mathrm{~h}$ per bed per day, the maximum daily bed capacity for 18 beds is 162 hours.

The ACU is open Monday to Friday and with 10 consultation periods per week corresponding to morning periods from $8 \mathrm{~h} 30$ to $12 \mathrm{~h}$ and afternoon periods from $13 \mathrm{~h} 30$ to $15 \mathrm{~h} 30$. There is no consultation on Friday afternoon, i.e. $B_{10}=0$. Chemotherapy sessions longer than 4 hours are assigned to morning periods, i.e. $x_{i t}=0, \forall i \in P / d_{i} \geq L_{t}=4 h, t \in P M$. The maximum bed capacity requirement of chemotherapy sessions planned for each afternoon is limited to $Q_{t}=18 \times L_{t}=72 \mathrm{~h}$.

There are 3 consultation boxes in the morning and 2 in the afternoon. One consultation box is dedicated to the intern. As a result, the 10 oncologists share the 14 consultation slots with 10 in the morning and 4 in the afternoon which meet the sufficient condition of Theorem 2 and ensure the feasibility of the medical planning problem. A consultation takes about 15 to 30 minutes depending on the skill of the physician. We assume that a physician can make until 14 consultations in the morning and 8 in the afternoon, i.e. $N_{t}=N_{t}{ }_{t}=14$, for all morning period $t, N_{t}=N^{\prime}{ }_{t}=8$, for all afternoon period $t$ except Friday afternoon, $N_{t}=N^{\prime}{ }_{t}=0$, for the Friday afternoon period $t$.

The elementary time unit is $\Delta=15$ minutes. The penalty factor $M$ of criterion (1) is selected to be large enough with $M=1000$ in order to avoid using extra consultation capacity whenever it is possible.

Numerical experiments are first conducted with data of the first two trimesters of year 2008. A time horizon of $W=12$ weeks is considered. The ACU delivered 2178 chemotherapy sessions for over 750 patients in the first trimester and 2084 chemotherapy sessions for 709 patients during the second trimester. Unfortunately, data collection turned out to be difficult. Injection times $d_{i}$ for about $5 \%$ of patients are missing and are generated by uniform random sampling from all known injections times. Five different problem instances for each trimester are generated and are denoted as $i_{-} 0, i \_1, i_{-} 2, i \_3$, and $i_{-} 4$ for instances of trimester $i$.

Additional numerical experiments will also be performed to assess the sensitivity with respect the number of patients. 


\subsection{Comparison of medical planning strategies}

This subsection compares three medical planning strategies: the current planning, the MIP strategy and the 3-stage approach. The current planning is the actual medical planning and patient assignment used by the ACU during the first two trimesters of year 2008. It is available from the field data. The criterion value of the current planning for each problem instance can be easily estimated from parameters of the problem instance and the MIP model. Note that the current planning strategy places each new patient in the first available time slot of the first week according to the medical planning. As a result, the medical planning and patient assignment are determined without taking into account impact on the bed capacity requirement.

The MIP strategy consists in solving directly the MIP model by means of a Branch and Bound algorithm with the commercial optimization engine ILOG CPLEX Concert 11 API for C++ running on a $2.4 \mathrm{GHz}$ Intel Core processor. We limit the computation time to 10 hours in order to be sure of having a good enough feasible solution. The computation time is very long because of significant symmetry of the problem and the large number of patients. For example, patients with the same protocol and the same referee oncologist and starting treatment in the same week are completely interchangeable.

The three-stage approach is implemented by using CPLEX to solve all optimization problems including $M I P(y), L B M I P(y), M I P^{A M}, M I P^{P M}$, and the existence of a solution of linear relaxation of the system defined by (2)-(11) and (17).

We first compare the bed capacity requirements of the current planning and the MIP strategy in order to show the benefit of optimization. Figure 3 illustrates the daily total bed capacity requirements, also called bedload in this section, of the two strategies for one problem instance of the first trimester of year 2008 (problem instance 1_4 in Table 3). The current planning strategy leads to large variations of bed capacity requirements (see for example the last week of the trimester), the peak bed capacity requirement is about $160 \mathrm{~h}$ and the lowest bed capacity requirement is less than $40 \mathrm{~h}$. Further the bed capacity requirement frequently approaches the theoretical maximal daily capacity of $162 \mathrm{~h}$. This is the root cause of the bed crisis observed in practice. For days of high bed capacity requirement, the ACU faces stressed medical staff, unsatisfied patients as the high bed capacity requirement results in long waiting times, and heavy workload of the pharmacy to prepare drugs. On the other side, for days of light bed capacity requirement, medical staff and beds are most often left unused.

Compared with the current planning, the MIP strategy is able to better balance the daily bed capacity requirement. Further, extra consultation capacity is used in the current planning while it is not used in the MIP strategy. Unfortunately the computation time of 10 hours is too long for a real life application. 


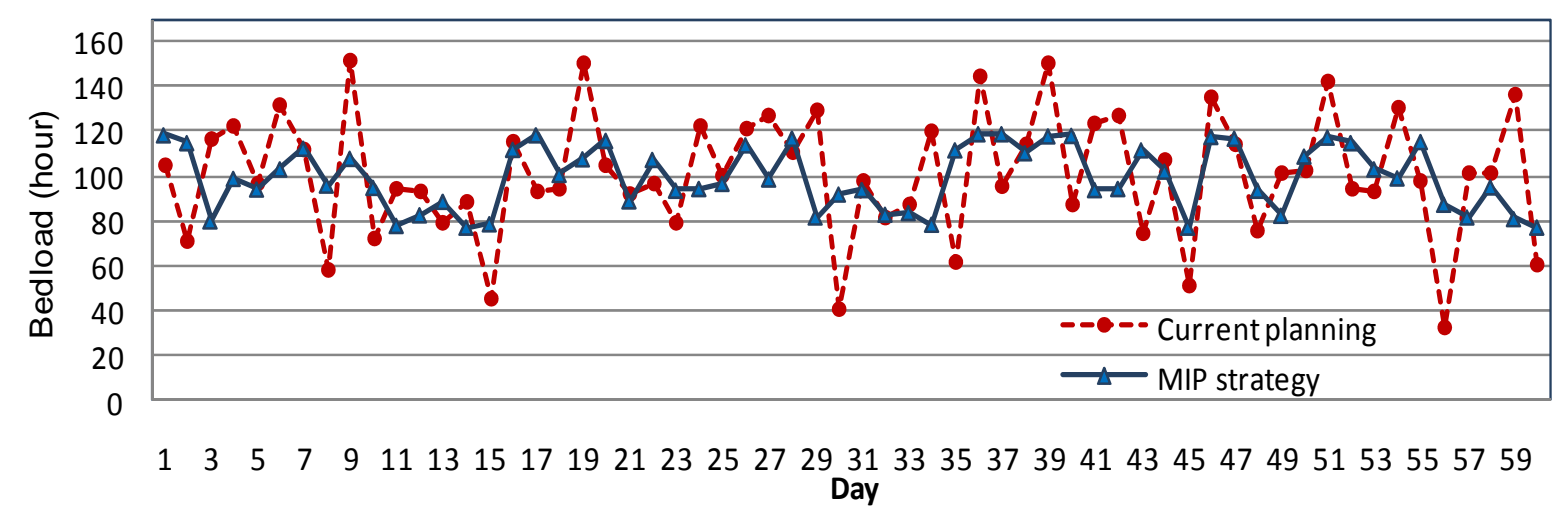

Figure 3: MIP strategy vs current planning.

Table 3: Bedload for $1^{\text {st }}$ and $2^{\text {nd }}$ trimesters of year 2008

\begin{tabular}{|c|c|c|c|c|c|c|c|c|c|c|c|c|c|c|}
\hline \multirow{2}{*}{ Instance } & \multicolumn{4}{|c|}{ Current planning } & \multicolumn{5}{|c|}{ MIP } & \multicolumn{5}{|c|}{ 3-stage approach } \\
\hline & Min & Max & Obj & extra & Min & Max & Obj & extra & CPU & Min & Max & Obj & extra & CPU \\
\hline $1 \_0$ & 38.5 & 162.2 & 835.5 & 1 & 67 & 130 & 260 & 0 & 36000 & 74.5 & 130 & 113.7 & 0 & 425 \\
\hline 1_1 & 32.5 & 159.2 & 801 & 1 & 67.7 & 129 & 249.5 & 0 & 36000 & 78.5 & 130.7 & 226.2 & 0 & 183 \\
\hline $1 \_2$ & 32.5 & 167.75 & 869.25 & 1 & 63 & 148.7 & 368.2 & 0 & 36000 & 67 & 141.5 & 132 & 0 & 357 \\
\hline $1 \_3$ & 31.25 & 155.5 & 791 & 1 & 63.5 & 127.7 & 240 & 0 & 36000 & 68.2 & 129.5 & 169.2 & 0 & 241 \\
\hline 1_4 & 67.8 & 162.3 & 1225 & 2 & 70.2 & 122 & 238 & 0 & 36000 & 71.5 & 119.2 & 100.7 & 0 & 532 \\
\hline $2 \_0$ & 41.75 & 146 & 698.7 & 0 & 65 & 107.5 & 140 & 0 & 36000 & 75.5 & 108 & 107.7 & 0 & 191 \\
\hline 2_1 & 42.25 & 151 & 726.5 & 0 & 71.5 & 105 & 92.5 & 0 & 36000 & 61.5 & 106 & 94.5 & 0 & 297 \\
\hline $2 \_2$ & 43.25 & 142 & 719 & 0 & 63.5 & 122.2 & \begin{tabular}{|l|}
382.2 \\
\end{tabular} & 0 & 36000 & 75.5 & 122 & 144 & 0 & 271 \\
\hline $2 \_3$ & 48.75 & 152.5 & 693.7 & 0 & 46 & 114.5 & 311.2 & 0 & 36000 & 70 & 119.5 & 176.2 & 0 & 568 \\
\hline $2 \_4$ & 42.25 & 138.5 & 696.2 & 0 & 62 & 112.5 & 191.5 & 0 & 36000 & 64 & 115.5 & 109 & 0 & 457 \\
\hline
\end{tabular}

Table 3 compares the bedload balancing, extra consultation capacity and the criterion value (1) of the current planning, the MIP strategy and the three-stage approach. The first column gives the problem instance where 1_i (resp. 2_i) corresponds a problem instance of the first (resp. second) trimester. Columns Min and Max represent the minimum and the maximum daily bed capacity requirement (in hour) over the whole planning horizon of one trimester ( $W=12$ weeks). Column Extra gives the extra consultation capacity used. Column CPU gives the CPU time (in seconds). As the MIP model is determined with an elementary time unit $\Delta=15$ minutes, we divide the value of the criterion (1) by 4 in order to express the bedloads in hours instead of $\Delta$. As a result, Column Obj gives the criterion value (1) of each strategy after scaling.

From Table 3, for all problem instances, the 3-stage approach gives the best criterion value in a reasonable computation time of less than 10 minutes and does not use any extra consultation capacity. Compared with the current planning, MIP strategy balances much better the bed capacity requirement with less extra consultation capacity and hence smaller criterion value but requires very long computation time. 
Apart from the gain in extra consultation capacity, let us evaluate the gain in peak daily bed capacity requirement, i.e. the columns Max. Compared with the current planning, for the 10 problem instances, the 3 -stage approach reduces the peak daily bed requirement by $20 \mathrm{~h}$ to $45 \mathrm{~h}$ with an average gain of 30h. The MIP approach reduces the peak daily bed requirement by $19 \mathrm{~h}$ to $46 \mathrm{~h}$ with an average gain of $30.5 \mathrm{~h}$. The above gains should be compared with the maximum theoretical bed capacity of $162 \mathrm{~h}$ for 18 beds with a maximum usage of $9 \mathrm{~h}$ each. The bed capacity requirement balancing allows the partner hospital ICL to avoid the bed crisis and to provide its medical staff with smoothed workload. It also allows ICL to accept more patients.

Note that it seems that the MIP approach sometimes leads to lower peak bed capacity requirement than the 3-stage approach. However, detailed analysis of bed capacity requirements over the entire time horizon show that the 3-stage approach actually better balances bed capacity requirement in each week and hence leads to better criterion value.

Consider now the current medical planning for the first trimester 2008 for problem instance 1_4. There are three consultation boxes $A M 1$ to $A M 3$ in the morning and two $P M 1$ and $P M 2$ in the afternoon. Table 4 gives the current medical planning with the $I D$ of the oncologist scheduled for each time slot. In this medical planning, a consultation box is sometime shared by several physicians in the same time period. Such organization causes conflicts between oncologists. Often, the first oncologist cannot finish all planned consultations on time and the second oncologist and his patients have to wait. Table 5 gives the average number of patients consulted each weekday by each oncologist including those seen by the intern. Consultations within the medical planning are highlighted. Due to poor medical planning and poor patient assignment strategy, the consultation capacity of oncologists is often exceeded and oncologists have to consult outside their medical planning to meet demand.

Tables 6 and 7 give a medical planning and the assignment of patients generated by the three-stage approach for problem instance 1_4 of the $1^{\text {st }}$ trimester 2008. From Table 7, the bedload is not proportional to the number of patients. In this instance, the daily bedload is well balanced over the week but Monday and Tuesday have significantly larger number of patients than Friday. As Friday afternoon is closed for consultation, patients assigned to Friday morning often have long injection times which lead to high daily bedload of Friday.

Let us compare the current planning and the three-stage planning. Sharing of consultation box in the same time period is allowed in the current planning but forbidden in the three-stage planning. The intern is not always used in the current planning while it is in the three-stage planning. Consultations outside medical planning are allowed in the current planning and forbidden in the three-stage planning. 
Of course, some of the outside medical planning consultations are due to medical reasons that are not considered in the three-stage planning.

We now perform a sensitivity analysis with respect to the number of patients to evaluate the performance of the 3-stage approach. The problem instances are generated randomly and the current planning is not available. As a result, we only compare the MIP approach and the 3-stage approaches.

The problem instances are generated as follows. The number of oncologists, the number of consultation boxes, the consultation capacity and the planning horizon $W$ are the same as before. However the set of patients to consider is different and is generated randomly as follows. We first generate for each week the number of new incoming patients according to a Poisson distribution with mean $\lambda$, i.e. the average number of new patients per week. For each new incoming patient, his protocol and referee oncologist are generated from a sampling population of over 2000 patients that we derived from the ICL information system. More specifically, a patient is randomly picked from the sampling population and we assume that the new incoming patient follows exactly the same treatment protocol with the same referee physician. This sampling procedure helps to preserve the proportions of patients per pathology and the number of patients per physician, while including uncertainties.

Six values of the average number of new patients per week are considered with $\lambda=\{19 ; 21 ; 22 ; 23 ; 24$; $25\} .100$ problem instances are generated for each $\lambda$ which lead to 600 problem instances in all.

Table 8 compares the performances of the MIP approach and the 3-stage approach for the 600 problem instances. Column $\lambda$ gives the average number of new patients per week. Column "\#" gives the average number of patients over 100 instances. Columns Min and Max represent the average of minimum and the maximum daily bed capacity requirement (in hour) over 100 instances. Column Extra gives the average extra consultation capacity used. Column Obj gives the average of criterion value. Column CPU gives the average CPU time (in seconds). The CPU time for MIP approach is limited to 10 hours and not presented in Table 8 .

From Table 8, the 3-stage approach always gives better objective value in shorter time compared to 10 hours for the MIP approach. Also the 3-stage approach better balances bed capacity requirement and uses less extra consultation capacity. 
Table 4: Current medical planning

\begin{tabular}{|c|c|c|c|c|c|}
\hline Box & Mon & Tue & Wed & Thu & Fri \\
\hline AM1 & 0 & 4 & 3 & 4 & 2 \\
\hline AM2 & 1 & 1 & $1 / 7$ & 3 & 0 \\
\hline AM3 & 8 & 5 & intern & 9 & intern \\
\hline PM1 & $2 / 9$ & $6 /$ intern & 8 & 7 & \\
\hline PM2 & 3 & 5 & intern & intern & \\
\hline
\end{tabular}

Table 5: Number of patients consulted in current medical planning

\begin{tabular}{|c|c|c|c|c|c|}
\hline Oncologist & Mon & Tue & Wed & Thu & Fri \\
\hline 0 & 16.8 & 2.5 & 1.4 & 2.1 & 17.3 \\
\hline 1 & 11.9 & 9.1 & 10.7 & 3.2 & 7.5 \\
\hline 2 & 3.5 & 0.1 & 0.5 & 0.2 & 9 \\
\hline 3 & 6.2 & 0.1 & 14.3 & 11.5 & 1.3 \\
\hline 4 & 0.9 & 17.8 & 0.5 & 18.8 & 0.6 \\
\hline 5 & 0 & 10.5 & 0.7 & 0.6 & 0 \\
\hline 6 & 0 & 1 & 0.2 & 0 & 0 \\
\hline 7 & 0 & 0.3 & 4.3 & 6.5 & 0.3 \\
\hline 8 & 7.9 & 0.5 & 5.7 & 0.4 & 0 \\
\hline 9 & 0.4 & 0.4 & 0 & 2.6 & 0 \\
\hline Patient \#/day & 47.6 & 42.3 & 38.3 & 45.9 & 36 \\
\hline Total Bedload (h) & 111.36 & 108.42 & 101.41 & 130.4 & 100.6 \\
\hline
\end{tabular}

Table 6: Medical planning of the 3-stage approach

\begin{tabular}{|c|c|c|c|c|c|}
\hline Box & Mon & Tue & Wed & Thu & Fri \\
\hline AM1 & 0 & 2 & 1 & 3 & 0 \\
\hline AM2 & 8 & 4 & 7 & 1 & 5 \\
\hline AM3 & intern & intern & intern & intern & intern \\
\hline PM1 & 4 & 3 & 6 & 9 & \\
\hline PM2 & intern & intern & intern & intern & \\
\hline
\end{tabular}

Table 7: Number of patients consulted in the 3-stage approach

\begin{tabular}{|c|c|c|c|c|c|}
\hline Oncologist & Mon & Tue & Wed & Thu & Fri \\
\hline 0 & 14 & & & & 13.2 \\
\hline 1 & & & 14 & 14 & \\
\hline 2 & & 12.82 & & & \\
\hline 3 & & 8.42 & & 14 & \\
\hline 4 & 8.42 & 14 & & & \\
\hline 5 & & & & & 10.5 \\
\hline 6 & & & 1 & & \\
\hline 7 & & & 11.5 & & \\
\hline 8 & 13.42 & & & & \\
\hline 9 & & & & 3.2 & \\
\hline Intern & 12.7 & 12.92 & 12.2 & 10.6 & 9.2 \\
\hline patient \#/day & 48.54 & 48.16 & 38.7 & 41.8 & 32.9 \\
\hline Total Bedload (h) & 109.79 & 110.5 & 109.77 & 111.96 & 110.17 \\
\hline
\end{tabular}


Table 8: $M I P$ and 3-stage approaches with different patient arrival rate

\begin{tabular}{|c|c|c|c|c|c|c|c|c|c|c|}
\hline \multicolumn{3}{|c|}{ Patients } & \multicolumn{4}{|c|}{ MIP } & \multicolumn{4}{|c|}{ 3-stage approach } \\
\hline$\lambda$ & $\#$ & Min & Max & Obj & Extra & Min & Max & Obj & Extra & CPU \\
\hline 19 & 713 & 38 & 104.5 & 80 & 0 & 68.8 & 101.9 & 62.6 & 0 & 491 \\
\hline 21 & 740 & 40 & 107.8 & 242.5 & 0 & 71.3 & 105.6 & 108.3 & 0 & 532 \\
\hline 22 & 756 & 40.8 & 107.3 & 227.8 & 0.2 & 72.9 & 105.5 & 106.2 & 0 & 612 \\
\hline 23 & 773 & 55 & 125.8 & 462.5 & 0.7 & 75.6 & 108 & 234.1 & 0.2 & 711 \\
\hline 24 & 780 & 58.8 & 135.8 & 731.8 & 0.6 & 78.1 & 109.2 & 178.6 & 0.4 & 800 \\
\hline 25 & 792 & 67.5 & 140 & 725 & 0.9 & 76.1 & 107.5 & 210.9 & 0.5 & 830 \\
\hline
\end{tabular}

\section{CONCLUSION}

This paper considered the medical planning problem of oncology ambulatory cares in order to best balance the bed capacity requirement. We proposed a formal innovative mixed integer programming (MIP) model. The MIP model captures main features of oncology chemotherapy such as treatment protocols and patient-oncologist relationships. Numerical experiments with data collected from the field showed the ability of MIP-based approaches to significantly smooth the bed capacity requirements over time. For the 10 problem instances derived from field data, we were able to reduce for about 20 to $45 \mathrm{~h}$ the peak bed capacity requirement while the theoretical ACU bed capacity is $162 \mathrm{~h}$.

Future research can be pursued in several directions. One immediate extension is to investigate the benefit of postponing the arrival of new patients for more than one week. A second important research direction is the assignment of incoming patients to different periods of the week. The current work relies on Assumption A5 which assumes full knowledge of all patients and is hence not applicable for patient assignment that is done once a week. Another important research direction is the appointment scheduling for all patients having their chemotherapy sessions the same day. More detailed information is needed and relations with the drug preparation should be taken into account. The simulation of the patient flows together with all relevant operations management strategies is another important issue. It requires faithful modeling of patient flows and patient treatment plan changes. Another relevant issue is the medical planning and patient assignment for special weeks of 4 days. The drug preparation scheduling in the pharmacy is another relevant issue. Finally, the impact of the reorganization of the ambulatory care units on other inpatient care units is a difficult issue of research.

Finally, while this paper focuses on the daily bed capacity requirement, another related issue is the workload of oncologists which is actually highly unbalanced. The workload of an oncologist actually depends on the specialty of the oncologist and his/her reputation. It is unclear how to take into account the workload balancing of oncologists in the medical planning. 


\section{APPENDIX}

Proof of Theorem 1: The proof is done by reducing polynomially the BIN-PACKING problem to a special case of the medical planning problem. The BIN-PACKING problem can be defined as follows: given a finite set of items $U$, a size $s(u) \in \mathbb{Z}^{+}$for each $u \in U$, a positive integer bin capacity $G$, and a positive integer $K$, is there a partition of $U$ into disjoint sets $U_{1}, U_{2}, \ldots, U_{K}$ such that the sum of the sizes of the items in each $U_{i}$ is $G$ or less? Consider also the PARTITION problem defines as follows: given a finite set $V$, a size $s(u) \in \mathbb{Z}^{+}$for each $u \in V$, is there a subset $V^{\prime} \subseteq V$ such that $\sum_{u \in V^{\prime}} s(u)=\sum_{u \in V-V^{\prime}} s(u)$ ? PARTITION is known to be NP-complete ([7]).

First, BIN-PACKING with $G=\sum_{u \in U} s(u) / K$ is also NP-complete for each fixed $K \geq 2$ as any PARTITION instance can be polynomially transformed into an equivalent instance of BIN-PACKING for any fixed $K \geq 2$ in which the set $U$ of items contains $V$ plus $K-2$ items of size $G$ and $\sum_{u \in V} s(u) / 2=\sum_{u \in U} s(u) / K=G$. It is obvious that the PARTITION instance has a solution if and only if its equivalent BIN-PACKING instance has a solution.

For each instance of BIN-PACKING with $K=5$ and $G=\sum_{u \in U} s(u) / K$, we transform it polynomially into an instance of the medical planning problem defined as follows. There are $|U|$ patients with injection times $d_{u}=s(u)$, i.e. $P=U$. A horizon of one week $W=1$ is considered. Each weekday corresponds to a bin, i.e. $K=5$. There is one oncologist with consultation capacity $N_{t}=|U|$, a single consultation box, $B_{t}=1$ and the afternoon bed capacity $Q_{t}=\sum_{i \in P} d_{i}$. The question is whether there is a medical planning solution such that $J^{*}=0$, i.e.. $\bar{C}_{w}=\underline{C}_{w}$ and the bed capacity requirement is the same over the week.

If BIN-PACKING instance has a solution, then $\sum_{u \in U i} s(u)=G$ for each bin $U_{i}$ and assign all patients corresponding to bin $U_{i}$ to the day $j$ leads to a medical planning solution with $\bar{C}_{w}=\underline{C}_{w}$ and $J^{*}$ $=0$. The reverse is also true. Hence, each BIN-PACKING instance has a solution if and only if its corresponding medical planning problem has a solution. This together with the NP-completeness of the BIN-PACKING problem concludes the proof. 


\section{ACKNOWLEDGMENT}

The authors would like to thank all physicians and nurses at the ICL Centre for their contribution in this study. In particular, we are grateful to the valuable insights and cooperation of Lionel Roesch, quality responsible, Dominique Feld, head nurse and Dr Pierre FOURNEL, chief resident.

\section{REFERENCES}

[1] Bailey NTJ, "A study of queues and appointment systems in hospital outpatient departments with special reference to waiting times," J. R. Stat Soc, Ser B 14:185-199, 1952.

[2] N. Blay, J. Cairns, J. Chisholm, J. O’Baugh, "Research into the workload and roles of oncology nurses within an outpatient oncology unit," European Journal of Oncology Nursing, vol. 6 (2002) 6-12.

[3] T. Cayirli, and E. Veral, "Outpatient scheduling in health care: A review of literature," Production and Operations Management, vol. 12 (2003) 519-549.

[4] T. Cayirli, E. Veral and H. Rosen, "Designing appointment scheduling systems for ambulatory care services," Health Care Manage Science, vol. 9 (2006) 47-58.

[5] D. Conforti, F. Guerriero, and R. Guido, "Optimization models for radiotherapy patient scheduling," 4OR, 6 (2008) 263-278.

[6] G. Delaney, B. Jalaludin, E. Moylan, M. Barton, "The Development of a Model of Outpatient Chemotherapy Delivery- Chemotherapy Basic Treatment Equivalent," Clinical Oncology, vol. 14 (2002) 406-412.

[7] M. R. Garey and D. S. Johnson, Computers and Intractability; A Guide to the Theory of NPCompleteness, Freeman, San Francisco, 1979.

[8] D. Gupta and B. Denton, "Appointment scheduling in health care: Challenges and opportunities," IIE Transaction, Vol. 40 (2008) 800-819.

[9] K. J. Klassen, T. R. Rohleder, "Scheduling outpatient appointments in a dynamic environment," Journal of Operations Management, 14 (1996) 83-101.

[10] L. Liu, X. Liu, "Block appointment systems for outpatient clinics with multiple doctors," Journal of Operational Research Soc, 49 (1998) 1254-1259.

[11] L. Liu, X. Liu, "Dynamic and static job allocation for multi-server systems," IIE Transactions, 30 (1998) 845-854.

[12] M. E. Matta and S. S. Patterson, "Evaluating multiple performance measures across several dimensions at a multi-facility outpatient center," Health Care Manage Science, 10 (2007) 173194. 
[13] A. Mazier, X. Xie, "Scheduling Physician Working Periods of a Chemotherapy Outpatient Unit," Proc. 13th IFAC Symposium on Information Control Problems in Manufacturing (INCOM'09), 2009.

[14] A. Sadki, X. Xie, F. Chauvin, "Patients assignment for an oncology outpatient unit". Proc. IEEE Conf. Automation Science and Engineering (CASE 2010), Toronto, CA, 2010.

[15] P. Santibáñez, S. V. Chow, J. French, L. M. Peterman, S. Tyldesley, "Reducing patient wait times and improving resource utilization at British Columbia Cancer Agency's ambulatory care unit through simulation," Health Care Management Science, 12(4), 392-407, 2009.

[16] P. Schaus, P. V. Hentenryck, "Scalable Load Balancing in Nurse to Patient Assignment Problems," CPAIOR, LNCS 5547, pp. 248-262, 2009.

[17] S. Walczak, W. E. Pofahl, R. J. Scorpio, "A decision support tool for allocating hospital bed required acuity of care", Decision support systems, 34 (2002) 445-456.

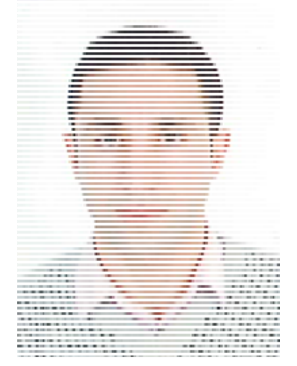

Abdellah SADKI received the B.S degree in industrial engineering from the Ecole Marocaine des sciences de l'Ingénieur, Rabat, Morocco, in 2006 and the M.S degree in Automatic Systems, Computers and Decisional from Paul SABATIER University of Toulouse, France, in 2008.

$\mathrm{He}$ is currently working toward the Ph.D. degree in industrial engineering at the Ecole Nationale Superieure des Mines de Saint Etienne (EMSE-SE), SaintEtienne, France. His research interests modelling, analysis and design methods to optimize health care delivery at an outpatient unit.

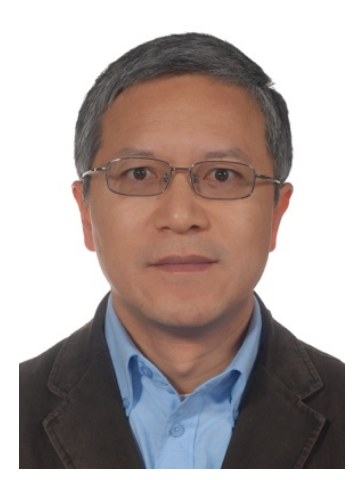

Xiaolan XIE received his Ph.D degree from the University of Nancy I, Nancy, France, in 1989, and the Habilitation à Diriger des Recherches degree from the University of Metz, France, in 1995.

Currently, he is a professor of industrial engineering and the head of Healthcare Engineering Department of the Center for Health Engineering, ENSM.SE, France. He is also a chair professor at the SJTU, Shanghai, China. Before Joining ENSM.SE, he was a Research Director at the Institut National de Recherche en Informatique et en Automatique (INRIA) from 2002 to 2005, a Full Professor at Ecole Nationale d'Ingénieurs de Metz from 1999 to 2002, and a Senior Research Scientist at INRIA from 1990 to 1999. His research interests include design, planning and scheduling, supply chain optimization, performance evaluation, and maintenance of manufacturing and healthcare systems. He is author/coauthor of over 200 publications including over 80 journal articles and five books. He has rich industrial application experiences with European industries. He is the French-leader for various EU-funded projects including FP6-IST6 IWARD on swarm robots for health services, FP6-NoE I*PROMS on intelligent machines and production systems, the FP5-GROWTH-ONE pro- 
ject for the strategic design of supply chain networks, the FP5- GRWOTH thematic network TNEE on extended enterprises.

He has been an associate editor on the conference editorial board of the IEEE Robotics and Automation Society, IEEE Transactions on Automation Science \& Engineering, IEEE Transaction on Automatic Control and IEEE Transactions on Robotics \& Automation. He has a Guest Editor of various special issues on health care engineering in IEEE Trans. Syst. Man \& Cybernetics, Annals of Operations Research (AOR, 2010), and Health Care Management Science (HCMS, 2009), and four other special issues on discrete event systems and manufacturing systems for IJCIM (2005), IJPR (2004, 2001), IEEE Trans. Robotics \& Automation (2001). He is general chair of ORAHS'2007, IPC chair of the IEEE Workshop on Health Care Management WHCM'2010 and IPC member for many other conferences.

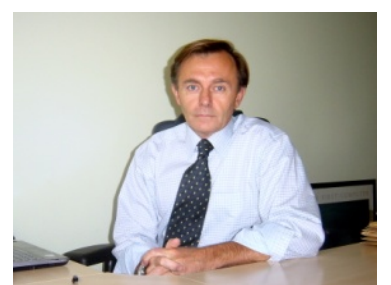

Franck CHAUVIN is medical oncologist, professor of public Health at Saint Etienne University and Loire cancer institute. He is the scientific director of a cancer prevention center in the French Rhône-Alpes region (centre Hygée) and head of a research team in the field of cancer prevention (primary, screening and patient education programs).

He is member of the French Public Health council (Haut Conseil de la santé Publique) and administrator of the French national cancer Ligue (ligue nationale contre le cancer). 